\title{
Vibration data acquisition system based on wireless transmission technology
}

\author{
Hao Zhang \\ China University of Mining and Technology , Beijing
}

Keywords: vibration data acquisition of wireless 、WiFi transmission、nRF905 、STM32F407

\begin{abstract}
The design plan to combine wireless control technology with wireless transmission technology and applied to the vibration data acquisition system, send the data collected by vibration sensor to the host computer through the WiFi wireless transmission. Let wireless transmission to replace the traditional wire data transmission, effectively solved the wiring inconvenience problem affect by the surrounding conditions, and effectively reduces the noise interference. Regard the footsteps vibration as the collection objects, based on the expected signal characteristics and the function of the system, optimize the design in hardware and software. In the aspect of hardware design, the nRF905 is chosen as the wireless communication module, STM32F407ZGT6 is chosen as the control chip of vibration data acquisition terminal, and the signal conditioning circuit, A/D conversion circuit was designed in consideration of the actual situation. What's more, two specific anti-interference measures was put forward. In the aspect of software design, main program and each part of the subroutine were designed respectively by the idea of modular design. And draw the corresponding program flow chart module as well. After complete the system overall design, also detailed debug the overall acquisition system and achieve the predetermined analysis effect of vibration signal acquisition. This system has been widely applied in the field of public safety and home security.
\end{abstract}

\section{Introduction}

The technology of data acquisition and transmission is very important for the social security, in the classification system of science and technology, data acquisition and transmission especially industrial vibration data acquisition has become an important part of public security field. According to the requirements of social development, the system combines the current popular wireless technology with vibration data acquisition in order to replace the traditional wired transmission, highlighting the wireless control mode's advantages relative to the wired connection, such as low cost, less interference in the process of transmission and high reliability. With the increasingly rapid society pace, the mobility of the population increase, the social structure is becoming more and more complex, the Home Furnishing security awareness is more and more strong in our mind. The system is expected to be used for wireless intelligent control Home Furnishing security system in order to meet the growing demand for security Home Furnishing. In addition, together the wireless technology with data acquisition system has high practicability, and it has great significance to realize remote control.

\section{The overall design scheme of vibration data acquisition system}

The new type of vibration data acquisition system designed in this paper is the integration of wireless control technology, WiFi wireless transmission technology and the vibration data acquisition, it is divided into three parts, namely the vibration data acquisition terminal, MCU control and WiFi wireless information transmission. Vibration data acquisition terminal conversion the weak voltage signal output from SW-520D vibration sensor. MCU control and data acquisition terminal is connected through wireless WiFi technology, that is to say all the control command and the collected vibration data are transmitted by WiFi technology. The flow charts are as follows: 


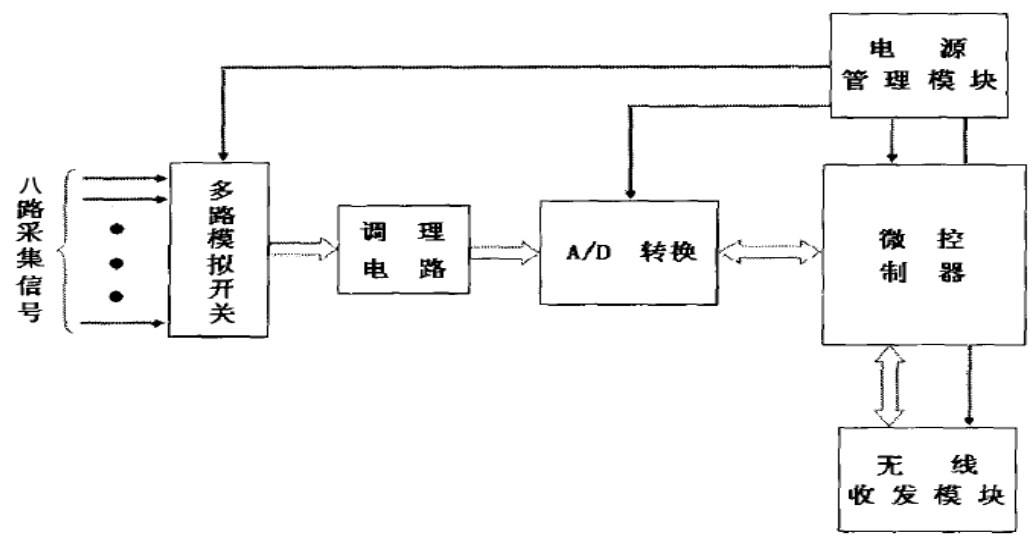

Part of vibration data acquisition

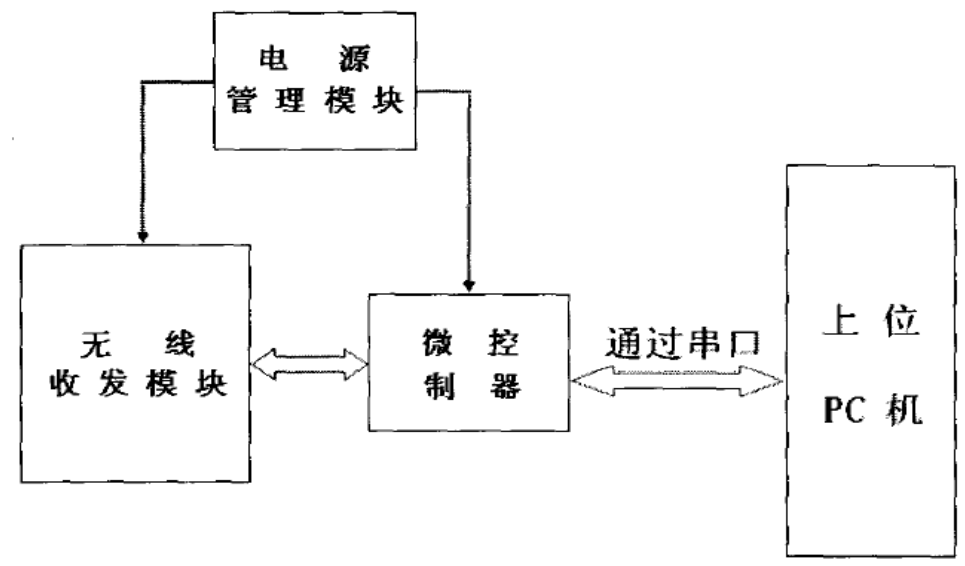

Part of the MCU control

\section{The hardware circuit design based on wireless transmission}

The core of this system is the hardware circuit design and performance of the new type of vibration data acquisition system depends largely on the circuit. The hardware design includes the key element selection ; the circuit principle diagram according to the overall design ; the system required a series of peripheral circuit such as conditioning circuit, A/D circuit and other related extension. After PCB board is completed, the hardware of the system should be checked, whether the hardware circuit welding is correct or there is a short-circuit.

The design of hardware circuit used ADS1274 as A/D converter, ADS1274 is a 4 channel multiplexing, 24 phase high performance, high speed, high precision, low power consumption, it also can achieve 18 bit resolution at the sampling frequency of 20KHz.ADS1274 has high resolution to the weak the vibration signal which meet the requirements of detection of weak vibration signals, suitable for vibration monitoring application. This system according to the actual requirements of detection of footsteps, set data output in serial mode after the completion of AD conversion which further simplified the connection between ADS1274 converter and STM32F407, thus improving the reliability design of AD conversion circuit. ADS1274 pin out diagram as shown in figure 


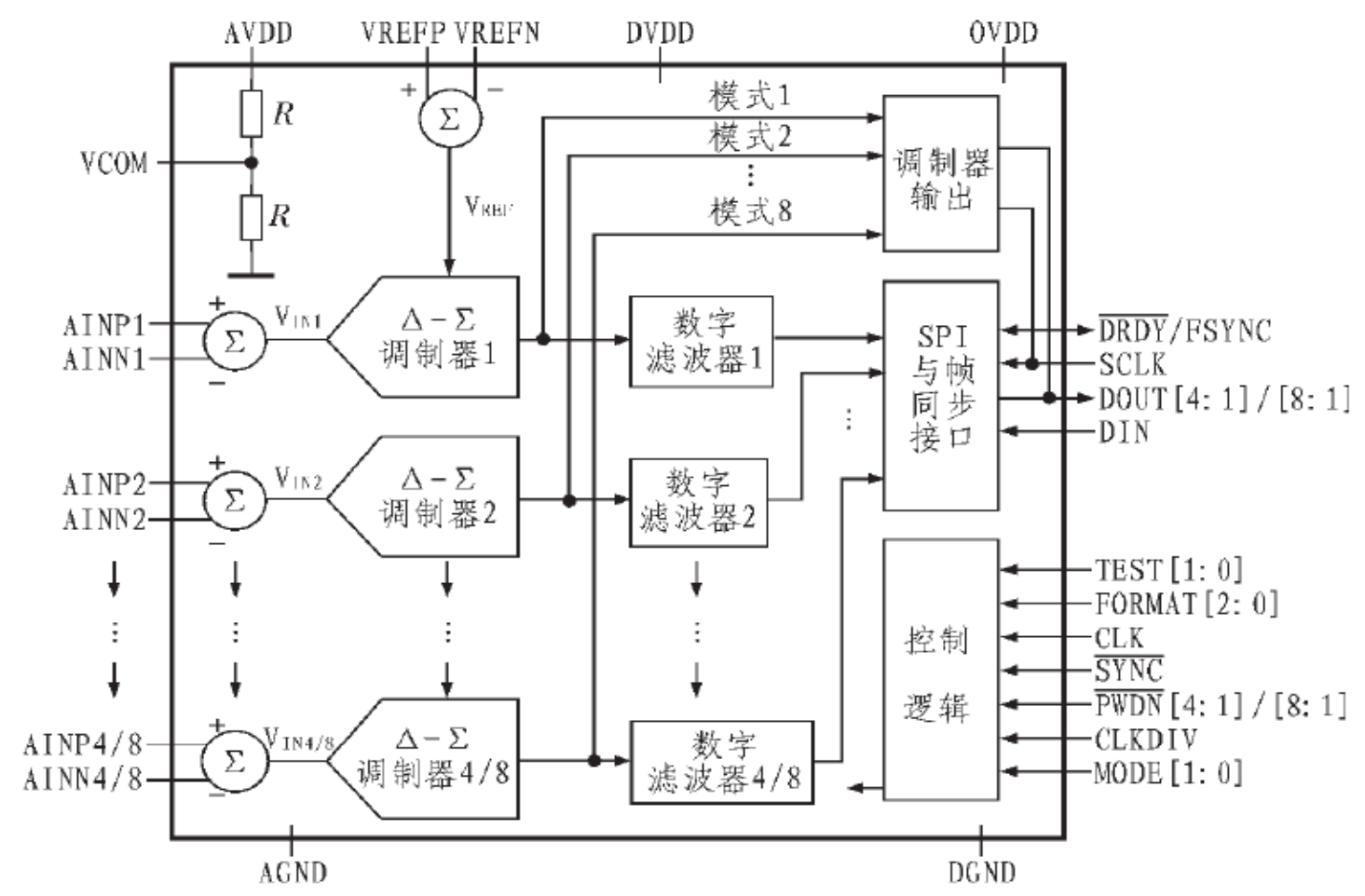

According to the actual requirements of footsteps vibration data acquisition, measurement range of vibration accelerometer should be $25 \mathrm{~g}$, in order to improve the sensitivity of the vibration sensor, the conditioning circuit is added in the hardware design. AD8628 is a element which include low cost ,high accuracy and low noise characteristics. It takes $2.7 \mathrm{~V}$ to $5 \mathrm{~V}$ single power supply, can also choose a dual power supply. The application principle of AD8628 operational amplifier in conditioning circuit design as shown in the figure

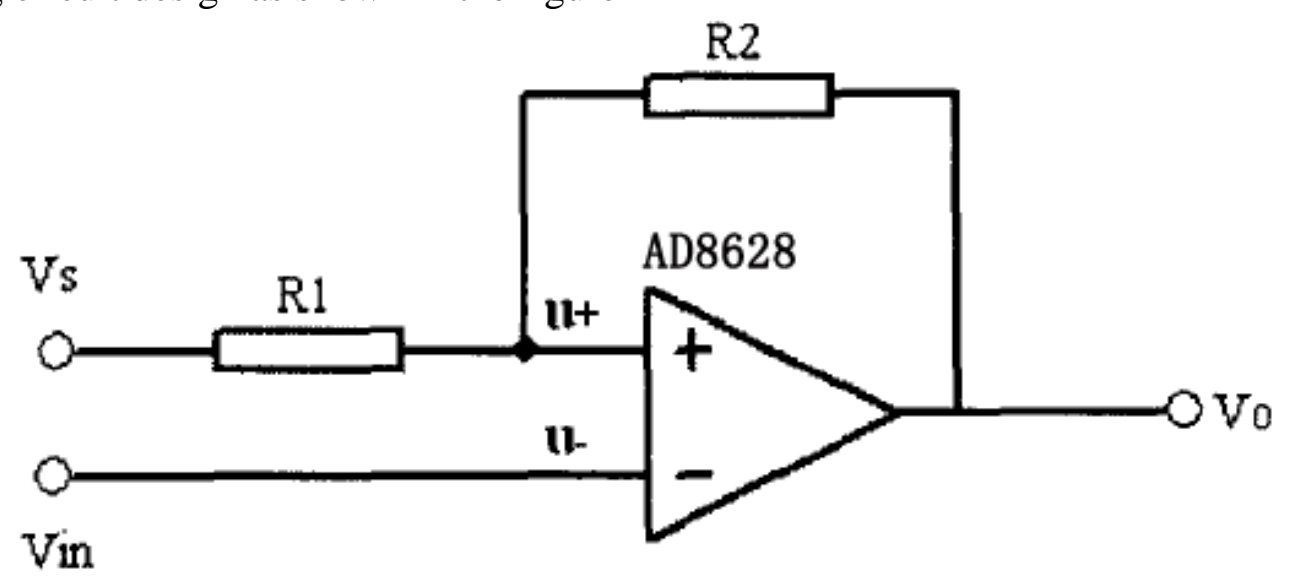

\section{The software design based on wireless transmission}

According to the overall design of the new data acquisition system needs and combined with the hardware circuit design has been completed, the software design can achieve the expected effect of the vibration acquisition system. The software design based on the modular design is divided into two parts, one of them is the vibration data acquisition part and the other is PC terminal control part. Using the modular design concept, block programming not only enhances the readability of the main program and subroutines but also convenient for the whole system simulation and debugging.

\subsection{The software design of the data collection terminal}

Initialization should begin after electrical work on PCB board, including basic configuration on serial port communication, write configuration words to nRF905, then send the actual running state 
of MCU through asynchronous traffic serial port to the STM32F407 control chip, the acquisition microcontroller will stay in waiting address query state. Microcontroller CPU continually send commands to the serial port of real-time query the current signs; microcontroller immediately began to receive instructions from the host PC serial port when CPU received the current position information, according to the different system instructions to complete the corresponding operation, and finally return to the corresponding state.

\subsection{The software design of the control part}

MCU should initialization first before the main program is running, and write configuration word to nRF905, let chip in the receiving state, then set the initial state for the "O" and open the interrupt response. After that, MCU will be uninterrupted query the real-time operation state,. When the microcontroller running state changes, STM32F407 will transfer to perform operation state to finish the operation command, the command and operation relationship are as follows:

The acquisition state: "acquisition" command, the MCU sends command to the data acquisition terminal and start work;

Stop state:" stop" commands, at this time acquisition will not take any single action.

\section{Debugging on the hardware and software of the data acquisition system}

The debugging of new wireless vibration control data acquisition system can be divided into two parts: software debugging and hardware circuit simulation. The software part debugging is compiled the existing procedures to detect the possible syntax error. Because the design of the software part adopts the module design process, so you can compile and detect for each sub module individually, debugging the integration system program after confirm each part is correct. Connect J-LINK simulator with the target board and take online simulation test is the hardware simulation. Debugging for the hardware simulation acquisition system can be divided into four parts:

1.Serial communication between microcontroller STM32F407ZGT6 and PC;

2 The voltage signal output after AD transform under the control of microcontroller in vibration data acquisition;

3. WiFi wireless communication protocol between the MCU control unit and the vibration data acquisition unit;

4.The overall detection of acquisition system

\section{Summary}

With the development of science and technology, data acquisition technology has gradually become an important way for people to obtain and receive information. In the modern time, wireless transmission technology is gradually replacing the wired transmission and become the main means of data transmission and host computer. Wireless transmission technology development has influenced the development of technology and modern people's daily life in many aspects, such as the layout of building automation, the family of intelligent wireless security etc.. This paper combined wireless control and wireless transmission technology with the vibration data acquisition system, take full consideration of the actual application. The wireless control acquisition system has broad application prospects in the field of intelligent furniture security, public safety monitoring etc. The main work of the research contents of this paper are summarized as follows:

1 Combine wireless control with WiFi wireless transmission technology, designed a new type of vibration data acquisition system hardware circuit,

The whole system take the high cost and low power consumption as the principle,

simplified circuit which is not only convenient to debug the system, but also reduce the cost of production.

2 Software design using $\mathrm{C}$ language, mainly including the main control program and MCU control program in the vibration data acquisition part.

Take Keil uvision4 software, XCOM serial assistant, J-LINK simulation device debugging.

3. Successfully install the whole system, achieve the desired design requirements of hardware 
and software, reached the expected requirements for simulation debugging the acquisition system.

\section{Reference}

[1] Yuan Xiguang. Handbook of sensor technology. Beijing: National Defence Industry Press, 2009

[2] Huang Zhiwei. Principle and application circuit design of radio frequency integrated circuit chip. Beijing: Beijing University of Technology press, 2004

[3] Dai Jia, Dai Weiheng. C language application programming example. Beijing: Electronic Industry Press, 2006.4

[4] Duan Xu section of the power supply circuit design manual. Shenyang: Liaoning science and Technology Press, 2002.8

[5] Huang Zhiwei. Design of wireless digital transceiver circuit. Beijing: Beijing Industry Press, 2003 